\title{
Analysis of a Porous-Inclined Slider Bearing Lubricated with Magnetic Fluid Considering Thermal Effects with Slip Velocity
}

Jay Pal Singh

jpsingh75@hotmail.com Advanced Institute of Tech. \& Managemen Depart. of Humanities \& Applied Sciences 121105 Palwal, Haryana, India

\author{
Naseem Ahmad \\ naseem_mt@yahoo.com \\ Jamia Millia Islamia \\ Department of Mathematics \\ 110025 New Delhi, India
}

A theoretical model of a porous-inclined slider bearing lubricated with magnetic fluid has been considered together with slip velocity boundary condition. Our aim is to study the influence of various dimensionless parameters arising out of the analysis of the model. By assuming the viscosity $\mu=\mu_{0} \exp \left[-\beta\left(t_{m}-t_{0}\right)\right]$ of magnetic fluid, the expressions for mean temperature and load capacity have been obtained. It has been observed that both mean temperature field and load capacity are the functions of slip parameter, magnetic parameter, thermal parameter and permeability parameter. The dependence of the mean temperature field as well as of load capacity on these parameters has been seen graphically.

Keywords: magnetic fluid, slip velocity, porous inclined-slider bearing, thermal effects, load capacity

\section{Introduction}

The lubrication behavior of different Newtonian and nonNewtonian fluids has been examined and analyzed by many researchers recently. It has been noted that the use of nonNewtonian fluids as lubricants becomes more important than the use of Newtonian fluids. It is a well-reported fact that the lubricants with stable suspensions of fine particles of insoluble solids having different material characteristics can be used as the viscosity index improver so that the viscosity variation with temperature may be prevented. It has been revealed that the behavior of such fluid is not Newtonian but non-Newtonian (Pal et al., 2002). For understanding frictional behavior and also for defining the physical conditions met by the oil in its passage through the conjunction of the disks, the knowledge of the temperature in the lubricant film is required. Due to the shearing of the oil in the film, the frictional heat exists (Crook, 1961). Because of the strong dependence of the viscosity on temperature, the temperature generated inside the film by the frictional heating causes an effective change in lubricant viscosity. Consequently, the viscosity must be assumed to be a function of temperature (Rodkiewicz and Dayson, 1974). Furthermore, in a heavily loaded system under hydrodynamic or EHD conditions, where high pressure exceeding $10^{7}$ dyne $\mathrm{cm}^{-2}$ is encountered, the lubricant viscosity is no longer insensitive to the pressure. Crook (1963) observed a 1000-fold increase in the viscosity ratio on raising the load from $2.5 \times 10^{7}$ to $19.7 \times 10^{7}$ dyne $\mathrm{cm}^{-1}$. Archard et al. (1961) and Dowson and Whitaker (1965), among the pioneers in this field, also emphasized the need to consider the variation in viscosity with pressure. It is therefore necessary to consider the effect of pressure and temperature on the lubricant viscosity (Cheng and Sternlicht, 1965; Kannel and Walowit, 1971; Rohde and Ezzat, 1974). In addition, the classical theory of hydrodynamic lubrication implicitly assumes that the lubricant behaves, essentially, as a Newtonian viscous fluid. This assumption, however, is not valid for fluids such as molten plastics, pulps, slurries, emulsions, greases, etc. These fluids exhibit non-Newtonian behavior and are widely used as lubricant in various lubrication flows. Therefore, the nonNewtonian behavior of the fluid in lubrication is also of considerable interest (Prasad et al., 1987).

The use of magnetic fluids has led to the development of many new energy devices and instruments. Magnetically cooled high-fidelity speakers, computer disc drives and semiconductors are already commercially available. Magnetic fluids are prepared by suspending ferromagnetic grains in non-magnetic nonconducting liquids such as dieters, kerosene, hydrocarbons and fluorocarbons. Ferromagnetic fluids, the non-conducting colloidal suspension of solid magnetic particles of sub-domain size in carrier liquids, are responsible for the development of several mechanical and electronic devices based on them (Ram and Verma, 1999). A typical magnetic fluid is prepared by suspending $\mathrm{Fe}_{3} \mathrm{O}_{4}$ particles of size $100 \AA$ which are coated with oleic acid in a diester. The most important property of magnetic fluids is that they can be made to adhere to any desired surface with the aid of magnets. When a magnetic field is applied, each particle experiences a force which causes it to move. When all the particles start moving, they cause the colloidal homogeneous suspension to move en masse (Agrawal, 1986).

Ochonski (2007) considered sliding bearings lubricated with magnetic fluids and suggested a new design of magnetic fluidbased sliding bearings. Patel (1980) studied the effect of slip velocity on the behavior of a squeeze film between two circular disks, the upper disk having a porous facing backed by solid housing, in the presence of a uniform transverse magnetic field. These investigations show that the effect of slip velocity reduces the load capacity of the bearing. Patel and Gupta (1983) used this slip condition at a porous boundary for hydrodynamic lubrication to analyze the problem of an inclined porous slider bearing. They suggested that minimization of the slip parameter is essential to increase the load capacity. Sinha et al. (2001) studied thermal effects in externally pressurized porous conical bearings with variable viscosity. Ahmad and Singh (2007a) used slip condition at a porous boundary for an inclined slider bearing without thermal effect. Further, Ahmad and Singh (2004b) investigated an inclined slider bearing with thermal effect.

In the present paper, magnetic fluid has been taken as a lubricant to study a porous-inclined slider bearing with thermal effect and slip velocity. The expressions for pressure and mean temperature have been derived. Subsequently, the formula for load has been obtained and the effect of various dimensionless parameters has been studied. Using Simpson's 1/3 rule the values of the mean temperature and load capacity for randomly chosen values of the magnetic parameter, slip parameter and thermal parameter have been computed to read the influence of these graphically. 


\section{Nomenclature}

$a \quad$ inlet-outlet ratio

$B_{0}=$ non-dimensional coefficient of temperature

$c_{p}=$ specific heat

$E=$ Eckert number

$h=$ dimensional film height

$\vec{H} \quad=$ external magnetic field

$\bar{h} \quad=$ non-dimensional film height

$h_{0}=$ minimum film thickness

$h_{1}=$ maximum film thickness

$k=$ porosity of the porous matrix

$\bar{k}=$ thermal conductivity

$l \quad=$ bearing wall thickness

$L \quad=$ bearing width

$\vec{M}=$ magnetization vector

$M^{*}=$ co-rotational derivative of $\vec{M}$

$M=$ magnitude of the magnetization vector

$\bar{M}=$ non-dimensional coefficient of viscosity

$p \quad=$ fluid pressure

$P \quad=$ non-dimensional fluid pressure

$P_{r} \quad=$ Prandtl number

$1 / \bar{s}=$ non-dimensional slip parameter

$s \quad=\alpha / k^{1 / 2}$, slip parameter

$t=$ temperature of the fluid

$t_{0}=$ temperature at $p=0$ (i.e., ambient pressure)

$t_{m}=$ mean temperature across the film thickness

$T_{m}=$ non-dimensional mean temperature

$T=$ non-dimensional temperature field

$u \quad=$ velocity component along the $x$-axis

$U=$ uniform sliding velocity component along the $x$-axis

$u_{0}=$ non-dimensional velocity component along the $x$-axis

$\vec{V} \quad=$ fluid velocity

$\bar{W}=$ non-dimensional load capacity

$x, y=$ Cartesian coordinates

$\bar{x}=$ non-dimensional $x$-coordinate

$\alpha=$ slip coefficient

$\alpha^{*}=$ material constant

$\beta=$ coefficient of temperature

$\bar{\beta}=$ permeability parameter

$\mu=$ coefficient of viscosity

$\mu_{0}=$ free space permeability

$\bar{\mu}^{*}=$ non-dimensional magnetic parameter

$\bar{\mu}=$ magnetic susceptibility

\section{Formulation of the Problem}

The configuration of a porous-inclined slider bearing lubricated with an incompressible magnetic fluid is shown in Fig. 1.

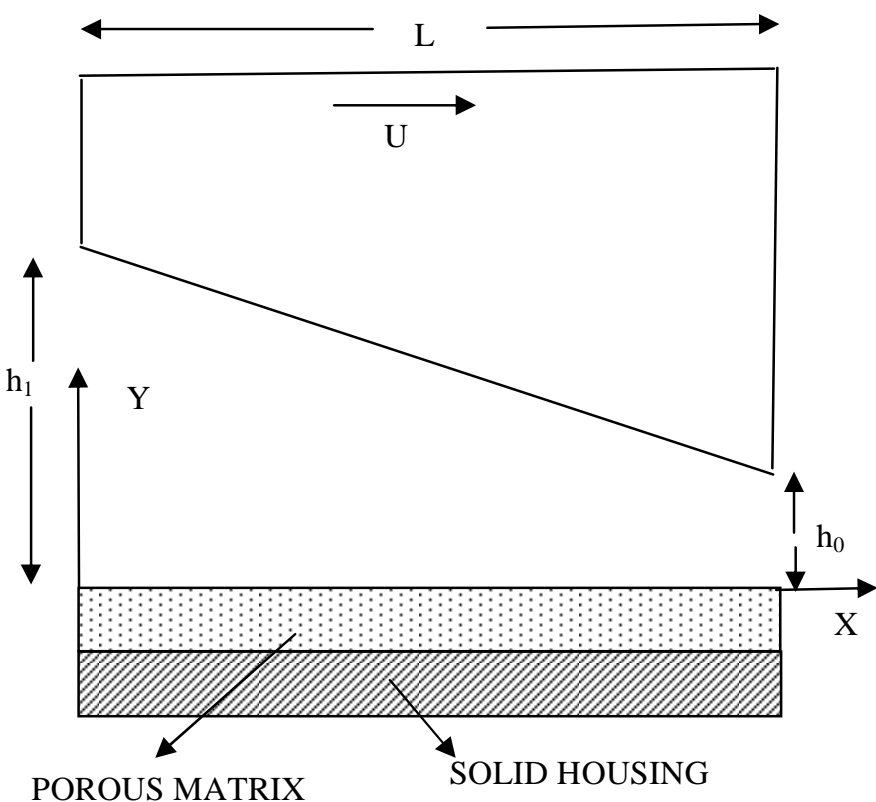

Figure 1. A porous-inclined slider bearing lubricated with magnetic fluid.

According to Agrawal (1986), the equations governing the flow of incompressible magnetic fluid in $D=\{(x, y): 0 \leq x \leq L, 0 \leq y \leq h\}$ are as follows:

$$
\begin{gathered}
\rho\left\{\frac{\partial \vec{V}}{\partial t}+(\vec{V} \cdot \vec{\nabla}) \vec{V}\right\}=-\vec{\nabla} p+\mu \vec{\nabla}^{2} \vec{V}+\bar{\mu}_{0}(\vec{M} \cdot \vec{\nabla}) \vec{H}+\rho \alpha^{*^{2}} \vec{\nabla} \times\left(\frac{\vec{M}}{M} \times M^{*}\right) \\
\vec{\nabla} \cdot \overrightarrow{\mathrm{V}}=0
\end{gathered}
$$

where $\vec{V}, p, \rho, \mu, \alpha^{*}, \vec{M}, M, M^{*}, \vec{H}$ and $\bar{\mu}_{0}$ are the fluid velocity, the pressure, the density, the coefficient of viscosity, a material constant, the magnetization vector, the magnitude of the magnetization vector, the co-rotational derivative of $\vec{M}$, the external magnetic field and the free-space permeability, respectively.

Because of magnetic fluid, we have the following relations based on Maxwell's equations:

$$
\begin{array}{ll}
\vec{\nabla} \times \overrightarrow{\mathrm{H}}=0, & \overrightarrow{\mathrm{H}}=-\vec{\nabla} \phi \\
\vec{\nabla} \cdot \vec{B}=0, & \vec{B}=\bar{\mu}_{0}(\vec{H}+\vec{M}) \\
\vec{M}=\bar{\mu} \vec{H} &
\end{array}
$$

where $\bar{\mu}$ is the magnetic susceptibility.

The equation of continuity in the porous region is given by

$$
\frac{\partial \bar{u}}{\partial x}+\frac{\partial \bar{v}}{\partial y}=0
$$

Under the normal conditions of lubrication theory and neglecting the self-field due to magnetization, we get the simplified forms of Eqs. (1) through (5) as: 


$$
\begin{aligned}
& \mu \frac{\partial^{2} u}{\partial y^{2}}-\frac{\partial}{\partial x}\left(p-\frac{\bar{\mu}_{0} \bar{\mu}}{2} H^{2}\right)=0 \\
& \frac{\partial u}{\partial x}+\frac{\partial v}{\partial y}=0 \\
& \frac{\partial}{\partial y}\left(p-\frac{\bar{\mu}_{0} \bar{\mu}}{2} H^{2}\right)=0 \\
& \bar{u}=-\frac{k}{\mu} \frac{\partial}{\partial x}\left(\bar{p}-\frac{\bar{\mu}_{0} \bar{\mu}}{2} H^{2}\right) \\
& \bar{v}=-\frac{k}{\mu} \frac{\partial}{\partial y}\left(\bar{p}-\frac{\bar{\mu}_{0} \bar{\mu}}{2} H^{2}\right)
\end{aligned}
$$

Substituting Eqs. (10) and (11) into Eq. (6), one obtains

$$
\frac{\partial^{2}}{\partial x^{2}}\left(\bar{p}-\frac{\bar{\mu}_{0} \bar{\mu}}{2} H^{2}\right)+\frac{\partial^{2}}{\partial y^{2}}\left(\bar{p}-\frac{\bar{\mu}_{0} \bar{\mu}}{2} H^{2}\right)=0
$$

The relevant boundary condition for the velocity field in the lubricant region is

$$
u=U \quad \text { at } \quad y=h
$$

where $U$ is the uniform sliding velocity component along the $x$-axis and $h$ is the dimensional film height.

As the slip velocity at the porous matrix is being introduced, the slip velocity due to Patel and Gupta (1983) is given by

$$
\frac{\partial u}{\partial y}=\frac{\alpha}{k^{\frac{1}{2}}} u \text { at } y=0
$$

where $k$ is the porosity of the porous matrix and $\alpha$ is the slip coefficient which depends on the structure of the porous material.

For pressure, the appropriate boundary conditions are

$$
p=0 \quad \text { at } \quad x=0, L
$$

where $L$ is the width of the bearing.

Solving Eq. (7) and applying the boundary conditions (13) and (14), we have the velocity component as

$$
u=\frac{y^{2}}{2 \mu} \frac{\partial}{\partial x}\left(p-\frac{\bar{\mu}_{0} \bar{\mu}}{2} H^{2}\right)+\left\{\frac{U-\frac{h^{2}}{2 \mu} \frac{\partial}{\partial x}\left(p-\frac{\bar{\mu}_{0} \bar{\mu}}{2} H^{2}\right)}{(1-s h)}\right\}(1-s y)
$$

where $s=\frac{\alpha}{k^{1 / 2}}$ is the slip parameter.

Using Eqs. (8), (12) and (15), one can get the Reynolds equation in the following form:

$$
\frac{\partial}{\partial x}\left(p-\frac{\bar{\mu}_{0} \bar{\mu}}{2} H^{2}\right)=\frac{\mu\{6 U h(2-s h)-12 A(1-s h)\}}{\left(12 k l-12 k l s h+4 h^{3}-h^{4} s\right)}
$$

where $A$ is the constant of integration and $l$ is the bearing wall thickness.

\section{Heat Transfer Problem}

We assume that the flow of lubricant is thermally active while surfaces are not. Under these assumptions, the energy equation is simplified to get

$$
\bar{k}\left(\frac{\partial^{2} t}{\partial y^{2}}\right)=-\mu\left(\frac{\partial u}{\partial y}\right)^{2}
$$

Let the viscosity $\mu$ be variable and it varies with temperature raised by frictional heat generated by flow of fluid having thermal conductivity $\bar{k}$. The expression $\mu$ due to Prasad et al. (1987) is

$$
\mu=\mu_{0} \exp \left[-\beta\left(t_{m}-t_{0}\right)\right]
$$

where $\mu_{0}$ and $t_{0}$ are, respectively, the viscosity coefficient and temperature when $p=0$ (ambient pressure). $\beta$ is the thermal coefficient and $t_{m}$ is the mean temperature across the film thickness $h$, which is defined as

$$
t_{m}=(1 / h) \int_{0}^{h} t d y
$$

The boundary conditions for the temperature field in the lubricant region are

$$
t=t_{0} \text { at } y=0 \text { and } y=h
$$

Introducing the following dimensionless variables:

$$
\begin{aligned}
& \bar{x}=\frac{x}{L}, \bar{y}=\frac{y}{h_{0}}, \quad \bar{h}=\frac{h}{h_{0}} \quad, \bar{M}=\frac{\mu}{\mu_{0}}, \bar{A}=\frac{12 A}{U h_{0}} \\
& u_{0}=\frac{u}{U}, P=\frac{p h_{0}^{2}}{\mu U L}, \quad T=\frac{t}{t_{0}} \quad, T_{m}=\frac{t_{m}}{t_{0}}, \bar{\beta}^{3}=\frac{12 k l}{h_{0}^{3}} \\
& P_{r}=\frac{\mu_{0} c_{p}}{\bar{k}}, E=\frac{U^{2}}{c_{p} t_{0}}, B_{0}=\beta t_{0}, \bar{\mu}^{*}=\frac{\bar{\mu}_{0} \bar{\mu} h_{0}^{2} L}{\mu U}, \bar{s}=s h_{0}
\end{aligned}
$$

where $c_{p}, \bar{h}, h_{0}, h_{1}$ and $\bar{\beta}$ are the specific heat, the nondimensional film height, the minimum film thickness, the maximum film thickness and the permeability parameter, respectively.

The non-dimensional forms of Eqs. (16), (18), (19) and (20) are

$$
\begin{aligned}
& u_{0}=\frac{\bar{y}^{2}}{2} \frac{\partial}{\partial \bar{x}}\left\{P-\frac{\bar{\mu}^{*}}{2} \bar{x}(1-\bar{x})\right\}+\left[1-\frac{\bar{h}^{2}}{2} \frac{\partial}{\partial \bar{x}}\left\{P-\frac{\bar{\mu}^{*}}{2} \bar{x}(1-\bar{x})\right\}\right] \frac{(1-\bar{s} \bar{y})}{(1-\bar{s} \bar{h})} \\
& \frac{\partial^{2} T}{\partial \bar{y}^{2}}=-\bar{M} P_{r} \cdot E\left(\frac{\partial u_{0}}{\partial \bar{y}}\right)^{2} \\
& \overline{\mathrm{M}}=\exp \left\{-\mathrm{B}_{0}\left(\mathrm{~T}_{\mathrm{m}}-1\right)\right\}
\end{aligned}
$$




$$
T_{m}=(1 / \bar{h}) \int_{0}^{\bar{h}} T d \bar{y}
$$

where $P, \bar{\mu}^{*}, 1 / \bar{s}, \bar{M}, P_{r}, E, B_{0}, T_{m}$ and $P_{r} . E$ are, respectively, the non-dimensional fluid pressure, magnetic parameter, slip parameter, coefficient of viscosity, Prandtl number, Eckert number, coefficient of temperature, mean temperature and thermal parameter.

Using Eqs. (22), (23) and boundary conditions $T=1$ at $\bar{y}=0$ and at $\bar{y}=\bar{h}$, we obtain the temperature field

$$
\begin{aligned}
& T=1-\bar{M} P_{r} \cdot E\left[\frac{\bar{y}^{4} \phi^{2}}{12}+\frac{\bar{y}^{2} \bar{s}^{2}\left(1-\frac{\bar{h}^{2} \phi}{2}\right)^{2}}{2(1-\bar{s} \bar{h})^{2}}-\frac{\bar{y}^{3} \phi \bar{s}\left(1-\frac{\bar{h}^{2} \phi}{2}\right)}{3(1-\bar{s} \bar{h})}\right] \\
& +\frac{\bar{y} \bar{M} P_{r} \cdot E}{\bar{h}}\left[\frac{\bar{h}^{3} \phi^{2}}{12}+\frac{\bar{h}^{2} \bar{s}^{2}\left(1-\frac{\bar{h}^{2} \phi}{2}\right)^{2}}{2(1-\bar{s} \bar{h})^{2}}-\frac{\bar{h}^{3} \phi \bar{s}\left(1-\frac{\bar{h}^{2} \phi}{2}\right)}{3(1-\bar{s} \bar{h})}\right]
\end{aligned}
$$

where $\phi=\frac{\partial}{\partial \bar{x}}\left\{P-\frac{\bar{\mu}^{*}}{2} \bar{x}(1-\bar{x})\right\}$

Putting this value of $T$ into Eq. (25) and on simplifying, we obtain mean temperature as

$$
T_{m}=1+\frac{\bar{M} P_{r} \cdot E}{240}\left\{6 \bar{h}^{4} \phi+\frac{5 \bar{h}^{2} \bar{s}^{2}\left(4+\bar{h}^{4} \phi^{2}-\bar{h}^{2} \phi\right)}{(1-\bar{s} \bar{h})^{2}}-\frac{5 \bar{h}^{3} \bar{s} \phi\left(2-\bar{h}^{2} \phi\right)}{(1-\bar{s} \bar{h})}\right\}
$$

The dimensionless form of Eq. (17) is

$$
\frac{\partial P}{\partial \bar{x}}-\frac{\bar{\mu}^{*}(1-2 \bar{x})}{2}=\left\{\frac{\frac{1}{\bar{s}}(12 \bar{h}-\bar{A})+\bar{h}(\bar{A}-6 \bar{h})}{\frac{1}{\bar{s}}\left(\bar{\beta}^{3}+4 \bar{h}^{3}\right)-\bar{h}\left(\bar{\beta}^{3}+\bar{h}^{3}\right)}\right\}
$$

where the film thickness $\bar{h}(\bar{x})$ of the inclined slider bearing is given by $\bar{h}(\bar{x})=a-(a-1) \bar{x}$, where $a=h_{1} / h_{0}, 0 \leq \bar{x} \leq 1$.

In particular, we take the inlet-outlet ratio

$$
a=h_{1} / h_{0}=2 \Rightarrow \bar{h}(\bar{x})=2-\bar{x}=2
$$

To compute load capacity, we use the formula

$$
\bar{W}=\int_{0}^{1} P d \bar{x}=-\int_{0}^{1} \bar{x} \frac{d P}{d \bar{x}} d \bar{x}
$$

Now, using Eqs. (27) and (29), we obtain load capacity of the bearing as

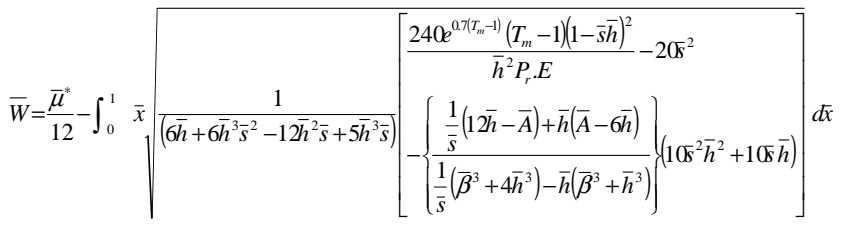

\section{Discussion and Results}

On the basis of the analysis and computation carried out for this problem, we recommended the following findings:

1. Figure 2 is a graph of mean temperature versus permeability parameter. It is seen that the mean temperature of the bearing increases as the permeability parameter increases. It has been noticed that mean temperature is increasing exponentially for $\bar{\beta}>0$.

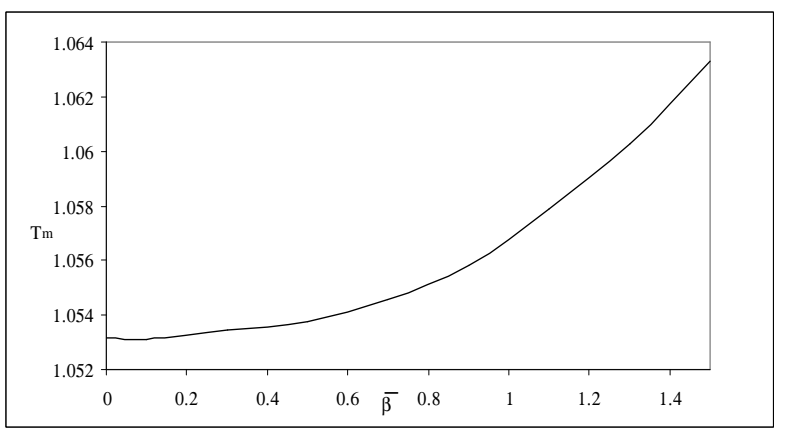

Figure 2. Mean temperature vs. permeability parameter at $1 / \bar{s}=3, P_{r} \cdot E=1.2, \bar{x}=0.6$.

2. Figure 3 shows a graph of mean temperature versus slip parameter. As the slip parameter increases, the mean temperature decreases. Physically, introduction of slip velocity reduces frictional between fluid and boundary due to which production heat may be reduced. This results in a fall in temperature.

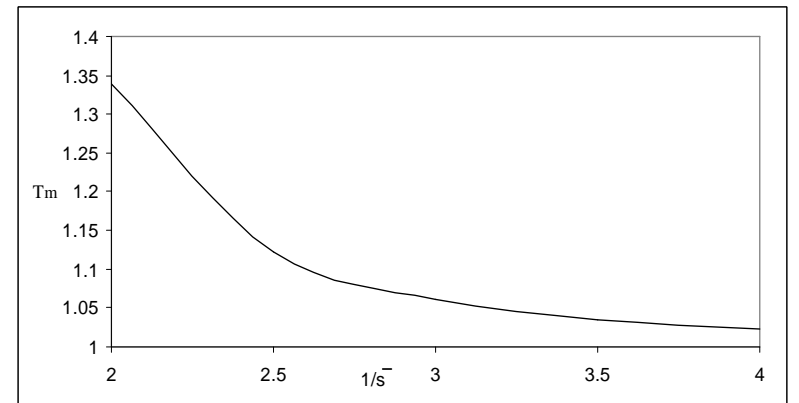

Figure 3. Mean temperature vs. slip parameter at $\bar{\beta}=1.3, P_{r} . E=1.2, \bar{x}=0.6$.

3. Figure 4 shows a graph of mean temperature versus thermal parameter. According to this graph, the mean temperature increases with the increase of the thermal parameter. The graph recommends the linear relation between $T_{m}$ and $P_{r}$.E. This result supports the statement of Hughes and Yong (1966). 


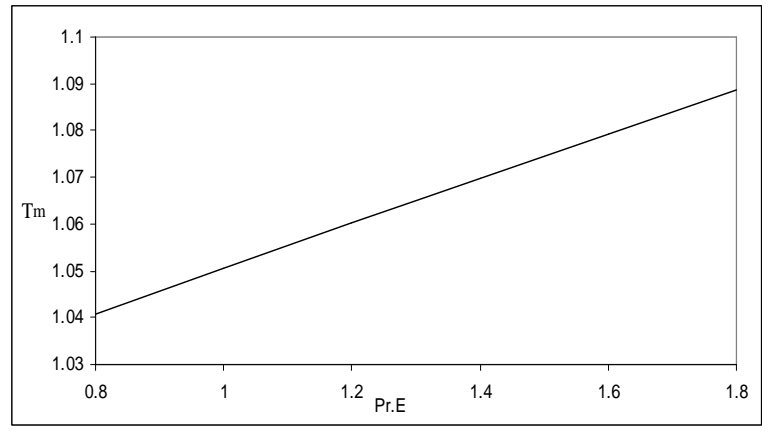

Figure 4. Mean temperature vs. thermal parameter at $1 / \bar{s}=3, \bar{\beta}=1.3, \bar{x}=0.6$.

4. Figure 5 clarifies the behavior of mean temperature against magnetic parameter. According to this graph, mean temperature increases with the increase of the magnetic parameter. The figure shows that $T_{m}$ is the linear function of $\bar{\mu}$.

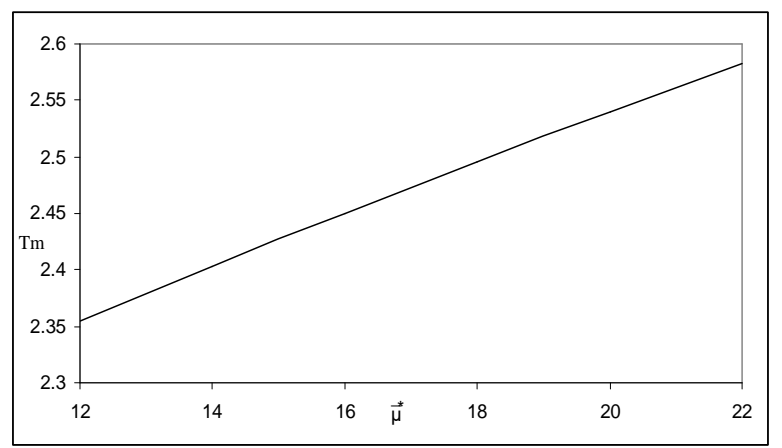

Figure 5. Mean temperature vs. magnetic parameter at $1 / \bar{s}=3, P_{r} E=1.2, \bar{x}=0.6$.

5. In Fig. 6, the slip parameter and the thermal parameter have been fixed and a graph of load capacity versus permeability parameter has been drawn to read the influence of the magnetic parameter. According to this graph, the impact of the permeability parameter is almost nil. It has also been noted that as the magnetic parameter increases, the load capacity also increases. This trend of dependence of load capacity on the magnetic parameter is also supported by the researches done by Agrawal (1986) and Ahmad and Singh (2007a).

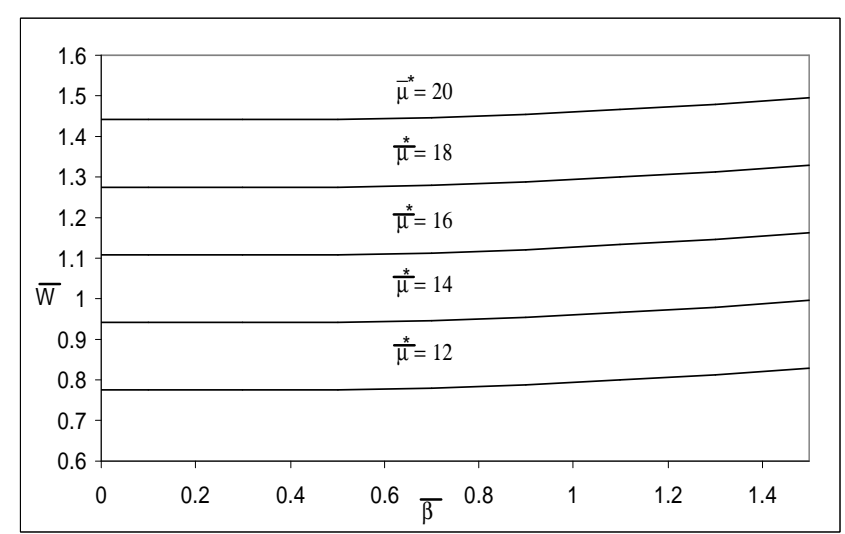

Figure 6. Load capacity vs. permeability parameter for different values of the magnetic parameter at $1 / \bar{s}=3, P_{r} . E=1.2$.

J. of the Braz. Soc. of Mech. Sci. \& Eng.
6. In Fig. 7, a graph of load capacity versus slip parameter has been plotted for different values of the magnetic parameter, while the permeability and thermal parameters are taken as constant. It is seen that the influence of the slip parameter is not remarkable, but the magnetic parameter boosts the load capacity.

\begin{tabular}{|c|c|c|c|c|c|c|}
\hline $\left.\begin{array}{l}1.6 \\
1.5\end{array}\right]$ & \multicolumn{5}{|c|}{$\mu^{*}=20$} & \\
\hline 1.4 & \multicolumn{5}{|c|}{$\mu^{*}=18$} & \\
\hline 1.2 & \multicolumn{5}{|c|}{$\mu^{*}=16$} & \\
\hline$\overline{\mathrm{W}}_{1}^{1.1}$ & \multicolumn{5}{|c|}{$\mu^{ \pm}=14$} & \\
\hline 0.9 & \multicolumn{5}{|c|}{$\frac{*}{\mu}=12$} & \\
\hline 0.7 . & & & & & & \\
\hline \multirow{2}{*}{2.5} & & & & & & \\
\hline & 2.75 & 3 & $1 / \bar{s}^{3} 3.25$ & 3.5 & 3.75 & 4 \\
\hline
\end{tabular}

Figure 7. Load capacity vs. slip parameter for different values of the magnetic parameter at $P_{r} . E=1.2, \bar{\beta}=1.3$.

7. Figure 8 exhibits the variation of the load capacity with respect to the thermal parameter as well as the magnetic parameter. It is concluded that the load capacity decreases slightly when the thermal parameter increases from 0.8 to 1.0 , and then it is almost constant beyond that. Therefore, the load capacity of the bearing is optimized when the thermal parameter is 0.8 .

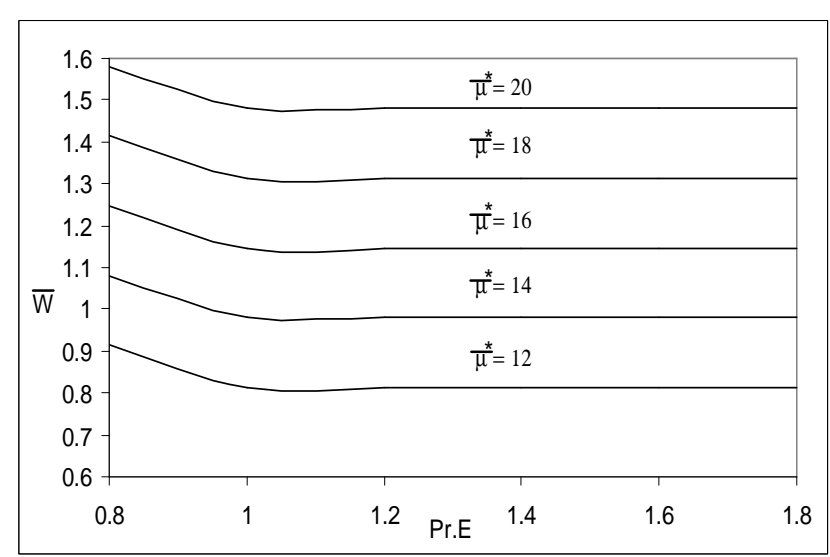

Figure 8. Load capacity vs. thermal parameter for different values of the magnetic parameter at $1 / \bar{s}=3, \bar{\beta}=1.3$.

\section{Conclusions}

A theoretical model of lubrication theory has been analyzed where magnetic fluid has been considered as lubricant with slip velocity effects. It has been recommended that permeability of the porous matrix accelerates mean temperature while slip velocity decelerates it. It has been concluded that mean temperature increases linearly with the thermal parameter.

The load capacity $\bar{W}$ has been studied in respect of the slip parameter, permeability parameter and thermal parameter. We see that the effect of slip and permeability parameters is not remarkable 
on load capacity $\bar{W}$ while the thermal parameter $P_{r} E$ has an influence within $0.8<P_{r} . E<1.0$ only.

The magnetic parameter boosts the load capacity $\bar{W}$ of the slider bearing.

\section{Acknowledgement}

The authors are thankful to Mr. Shivhari Dikshit, Assistant Professor in English, AITM, Palwal, who helped in improving the English language of this paper.

\section{References}

Agrawal, V.K., 1986, "Magnetic-fluid based porous inclined slider bearing”, Wear, Vol. 107, pp. 133-139.

Ahmad, N. and Singh, J.P., 2007a, "Analysis of a porous inclined slider bearing lubricated with a magnetic fluid considering slip velocity", Proc. IMechE Part N: J. Nanoengineering and Nanosystems, Vol. 221(N3), pp. 81-85.

Ahmad, N. and Singh, J.P., 2004b, "Magnetic fluid based porous inclined slider bearing considering thermal effects", Proc. $2^{\text {nd }}$ BSME-ASME International Conference on Thermal Engineering, Vol. II, pp. 1019-1025.

Archard, G.D., Gair, E.C., and Hirst, W., 1961, "The EHD lubrication of rollers", Proc. Roy. Soc. London, Ser. A262, 51, pp. 51-65.

Cheng, H.S., and Sternlicht, B., 1965, "A numerical solution for the pressure, temperature and film thickness between two infinitely long lubricated rolling and sliding cylinders under heavy load", ASME Trans. Ser. D., 87(3), pp. 695-707.

Crook, A.W., 1961, "The lubrication of rollers -III. A theoretical discussion of friction and the temperature in the oil films", Philos. Trans. Roy. Soc. London, Ser. A, Vol. 254, pp. 237-258.
Crook, A.W., 1963, "The lubrication of rollers IV. Measurement of friction and effective viscosity", Philos. Trans. R. Soc. London, Ser. A, Vol 255, pp. 281-312.

Dowson, D., and Whitaker, A.V., 1965, "The isothermal lubrication of cylinders", ASLE Trans., Vol. 8, pp. 224-234.

Hughes, W.F. and Yong, F.J., 1966, "The Electromagnetodynamics of fluid", $45 \mathrm{p}$.

Kannel, J.W., and Walowit, J.A., 1971, "Simplified analysis for traction between rolling sliding EHD contacts", J. Lubr. Technol., Vol. 93, pp. 39-46.

Pal, A.K., Das, N.C., and Chaudhary, R., 2002, "A Study of load capacity of finite slider bearings lubricated with couple stress fluids considering thermal effects", Indian J. Pure and Appl. Maths, 33(10), pp. 1529-1540.

Patel, K.C. and Gupta, J.L., 1983, "Hydrodynamic lubrication of a porous slider bearing with slip velocity", Wear, Vol. 85, pp. 309-317.

Patel, K.C., 1980, "The hydro magnetic squeeze film between porous circular disks with velocity slip", Wear, Vol. 58(2), pp. 275-281.

Prasad, D., Singh, P., and Sinha, P., 1987, "Thermal and squeezing effects in non-Newtonian fluid film lubrication of rollers", Wear, Vol. 119 , pp. $175-190$.

Ram, P. and Verma, P.D.S., 1999, "Ferro fluid lubrication in porous inclined slider bearing", Indian J. Pure and Appl. Maths, Vol. 30(12), pp. 1273-1281.

Rodkiewicz, C.M., and Dayson, C., 1974, "The thermally boosted oil lubricated sliding thrust bearing", J. Lubr. Technol., 96, pp. 322-329.

Rohde, S.M, and Ezzat, H.A., 1974, "A study of THD squeeze films", J. Lubr. Technol., Vol. 96, pp. 198-205.

Sinha, P., Chandra, P. and Bhartiya, S.S., 2001, "Thermal effects in externally pressurized porous conical bearings with variable viscosity", Acta Mechanica, Vol. 149, pp. 215-227.

Ochonski, W., 2007, "Sliding bearings lubricated with magnetic fluids", Industrial Lubrication and Tribology, 59/6, pp. 252-265. 